\title{
Superhydrophobic properties of cotton woven fabrics with conducting 3D networks of multiwall carbon nanotubes, MWCNTs
}

\author{
T. Makowski • D. Kowalczyk • W. Fortuniak • \\ D. Jeziorska $\cdot$ S. Brzezinski $\cdot$ A. Tracz
}

Received: 29 May 2014/ Accepted: 30 August 2014/Published online: 12 September 2014

(C) The Author(s) 2014. This article is published with open access at Springerlink.com

\begin{abstract}
This article presents the findings concerning the preparation and properties of cotton woven fabrics with a conductive network made of multiwall carbon nanotubes deposited on the fiber surface by the padding method. The next stage of treatment consisted of imparting superhydrophobic properties to the fabrics in solution with methyltrichlorosilane (MTCS) in a waterless medium. The tests performed show that the state of surface and water content in cotton fibers exerts a significant influence on the hydrophobic properties of the analyzed samples. In order to explain the differences in hydrophobic properties, the morphology of the cotton fabric surface was examined using samples with various water contents. The formation mechanism of MTCS coatings on cotton fabric has been proposed.
\end{abstract}

This article is dedicated to the memory of Prof. Adam Tracz, who passed away after a serious illness on 23 December 2013.

T. Makowski $(\bowtie) \cdot$ W. Fortuniak · D. Jeziorska ·

A. Tracz

Centre of Molecular and Macromolecular Studies Polish Academy of Sciences, Sienkiewicza 112, 90-363 Lodz, Poland

e-mail: tomekmak@cbmm.lodz.pl

D. Kowalczyk · S. Brzezinski

Textile Research Institute, Brzezinska 5/15, 92-103 Lodz, Poland
Keywords Cotton modification · Hydrophobization · Cotton superhydrophobic . Carbon nanotubes $\cdot$ Smart textile

\section{Introduction}

In recent years, superhydrophobic surfaces showing self-cleaning properties have aroused intense interest (Li et al. 2007). This feature results from the hierarchical combination of the micro- and nanostructure and a low surface energy of the material (Hosono et al. 2005). The most desirable from the practical point of view are transparent and durable superhydrophobic coatings on the surfaces of soft materials such as woven fabrics. The hydrophobicity effect in the case of hydrophilic fabrics can be obtained by depositing a dispersion or emulsion of the appropriate auxiliary agents, mostly in an aqueous medium, on their surfaces using the padding method. One of the main groups of such compounds consists of chlorosilane used for the preparation of organosilicon polymers (polysiloxanes) (Artus et al. 2006; Fadeev and McCarthy 2000), especially methylchlorosilanes containing various numbers of methyl groups (one, two or three) in a molecule depending on the modification degree and the type of product expected (Feng et al. 2002; Gao and McCarthy 2009; Gao et al. 2009; Xu et al. 2009).

A good hydrophobicity effect can be obtained by finishing textiles with the use of properly selected 
silanes and the sol-gel method (Hench and West 1990; Zha and Roggendorf 1991). The padding of textiles with aqueous or aqueous alcoholic solutions of sols synthesized from appropriately selected silanes, followed by drying and thermal cross-linking, under specified conditions, makes it possible to form very thin coatings $(100-200 \mathrm{~nm})$ on the surface of fibers. The coatings made in this way are durably combined with the fiber surface, being transparent and elastic, retaining the textile characteristics of the fabric. It should be noted that at the same time other teams in the world have produced a modified woven cotton fabric using modified multiwall carbon nanotubes (CNTs) (Alimohammadi et al. 2012; Alimohammadi 2013; Parvinzadeh Gashti and Almasian 2013). The authors showed a prominent role of stabilizing CNTs with the immersing method in textile production. However, the thus obtained material remains hydrophilic. At the same time, they impart additional specific features to the fabrics, including hydrophobicity.

Hydrophilic cotton fibers can obtain hydrophobic properties by their modification with CNTs. The basic condition for a positive result of such a modification is a uniform deposition of a thin CNT layer, which requires one to disperse CNTs in the aqueous padding bath to a large, possibly mono-particle degree. Owing to the three-dimensional structure of the carrier surface (cotton woven fabric), constituting a sort of "scaffolding," the deposited CNT network also acquires a 3D character. These "smart fabrics" are very interesting in terms of their utilization as lightweight, wearable, flexible conducting materials for a wide range of energy and environmental device applications, including electromagnetic shielding (AlShabib et al. 2012), antennas (Hertleer et al. 2009; Kennedy et al. 2009; Lee et al. 2011; Yang et al. 2007), microwave absorbers (Park et al. 2006), resistive heating elements (Fischer et al. 2011; Hamdani et al. 2013; Yang et al. 2010), ultracapacitors (Jost et al. 2011), batteries (Liu et al. 2012), water filtration (Schoen et al. 2010), microbial fuel cells (Xie et al. 2011) and printed electronics (Andersson et al. 2002).

The above observations have become the basis for undertaking studies on the hydrophobization of cotton woven fabrics covered with a multiwall CNT (MWCNT) layer. The electrically conducting woven fabrics were then hydrophobized by wet deposition of a properly selected methyltrichlorosilane (MTCS) in a waterless medium. Literature reports describe the effect of the reaction time and concentration of MTCS on the shape and size of the organosilicon nanostructures formed on the cellulose fiber surface (Shirgholami et al. 2013, 2011). Interestingly, in the course of investigations performed, we discovered that beside the factors mentioned above, a significant effect on the hydrophobicity of the modified woven fabrics is exerted by the relative humidity of environment/air $(\mathrm{RH})$ at which the tested samples were conditioned. To our knowledge, this dependence has not been described as yet in the related literature, which induced us to undertake systematic studies in this area, aimed at both maximization of the hydrophobicity effect obtained on cotton fabrics and an explanation of the MTCS coating mechanism. We expect that our findings could be used for the optimization of technological processes to impart both hydrophobic properties (water resistance) and photocatalytic selfcleaning (Ding et al. 2011) capabilities to textiles made of hydrophilic cotton fibers.

\section{Experimental}

Materials

\section{Cotton woven fabric}

A commercial woven fabric of $100 \%$ cotton fibers, with plain weave, a surface weight of $145 \pm 7 \mathrm{~g} / \mathrm{m}^{2}$ and a thickness of $0.36 \mathrm{~mm}$. Warp yarn: tex 25 , density of warp 295 threads $/ 10 \mathrm{~cm}$, weft yarn: tex 25 , density of weft 205 threads $/ 10 \mathrm{~cm}$. The woven fabric was prepurified/washed under industrial conditions and dried. To completely remove purification residues such as wax, fats and pectins, fabric samples were purified by extraction in turn with diethyl ether $(98 \%$ analytically pure, CHEMPUR) and anhydrous ethanol (analytically pure, CHEMPUR) for $2 \mathrm{~h}$ each. The fabrics were then additionally purified in boiling ethanol in a reflux condenser $(2 \mathrm{~h})$ followed by rinsing with double-distilled water and drying at a temperature of $120{ }^{\circ} \mathrm{C}$ for 30 min (Hock and Harris 1940).

\section{Hydrophobizing agent, solvents and dispersing agent}

- Hydrophobizing agent-MTCS ( $\geq 97 \%$, SigmaAldrich) 
- Solvent-anhydrous ethanol (analytically pure, CHEMPUR)

- Solvent-anhydrous toluene (CHEMPUR)

- Dispersing agent-SDS-sodiumlauryl sulfate ( $\geq 99 \%$, Carl Roth $\mathrm{GmbH}$ ).

\section{Carbon nanotubes}

The MWCNT Nanocyl NC-7000 (Nanocyl S.A., Belgium) with a diameter of $9.5 \mathrm{~nm}$, length of $1.5 \mu \mathrm{m}$, purity of $90 \%$ and a specific surface of $250-300 \mathrm{~m}^{2} / \mathrm{g}$.

\section{Preparation of aqueous dispersion of MWCNTs}

The nanotubes were dispersed in an aqueous solution of SDS (dispersing agent) (Vaisman et al. 2006). The concentration of MWCNTs in the aqueous dispersion was $0.5 \%$, and the ratio of MWCNTs to SDS was equal to $1: 3$. In order to obtain a homogeneous suspension, the whole was dispersed with the use of an ultrasonic sonotrode.

\section{Deposition of MWCNT dispersion on woven fabrics}

The aqueous dispersion of MWCNTs was deposited on dry fabric samples by the padding method using a laboratory double-roll padding machine with horizontally set squeezing rollers (Benz, Switzerland). The padding rate was $1 \mathrm{~m} / \mathrm{min}$ with a squeezing roller pressure amounting to $20 \mathrm{~kg} / \mathrm{cm}$ along the roller contact line. The saturated woven fabric was then dried at $100{ }^{\circ} \mathrm{C}$ for $15 \mathrm{~min}$. In order to remove the dispersing agent and excess nanotubes, the samples were rinsed several times in distilled water followed by drying in hot air at $120^{\circ} \mathrm{C}$ for $1 \mathrm{~h}$.

\section{Conditioning of fabric samples}

before the hydrophobization process

Before the hydrophobization of fabric samples, these were conditioned for $24 \mathrm{~h}$ at a temperature of $21^{\circ} \mathrm{C}$ in dessicators under vapors of saturated salt solutions at RH 40 and $70 \%$. A so-called "dry" sample at RH $0 \%$ was obtained by rinsing with dry nitrogen for $3 \mathrm{~h}$.
Hydrophobization of fabric samples in liquid phase by the immersing method in a waterless medium

Two types of samples were hydrophobized: a purified woven fabric $(\mathrm{CO})$ and purified cotton fabric with a deposited conductive network of multiwall CNTs $(\mathrm{CO}+\mathrm{MWCNTs})$. Samples were placed in a polypropylene vessel containing $0.5 \mathrm{M}$ solution of MTCS in anhydrous toluene ( $1 \mathrm{~g}$ of fabric per $100 \mathrm{ml}$ of solution). Reactions were run at a temperature of $25{ }^{\circ} \mathrm{C}$ for $1 \mathrm{~h}$. The samples were then rinsed in: toluene, ethanol, ethanol/water mixture (1:1) and water, followed by drying in hot air at a temperature of $120{ }^{\circ} \mathrm{C}$ for $15 \mathrm{~min}$.

Methods and apparatus

Surface morphology, content of the elements present in fibers and nanostructure formed

The surface and chemical compositions of the analyzed samples were determined using a scanning electron microscope (SEM, JEOL 5500) with energy dispersive $\mathrm{X}$-ray spectroscopy (EDS). Images were taken after the surface of samples had been coated with a gold layer (about $20 \mathrm{~nm}$ thick) by ion sputtering (JEOL JFC1200). To identify the internal structure of the nanoparticles formed, we used a transmission electron microscope (TEM, Tesla BS 512 with a YAG camera). The operating voltage was $90 \mathrm{kV}$ (tungsten filament).

Measurement of the water contact angle (WCA) of the analyzed samples

The water contact angles of the tested samples were measured using of a Rame-Hart NRL Goniometer (model 100-00-230) coupled with a camera and a light system. WCAs were determination by the author's Drop Program. Measurements were taken under normal conditions, locating a water drop with a volume of $5 \mu \mathrm{l}$ on the sample surface tested. The test was repeated for five different positions on each fabric surface for the determination of the average WCA values.

\section{Measurement of the sliding angle (SA)}

The sliding angles were measured using an apparatus of our own construction equipped with a glass mobile 

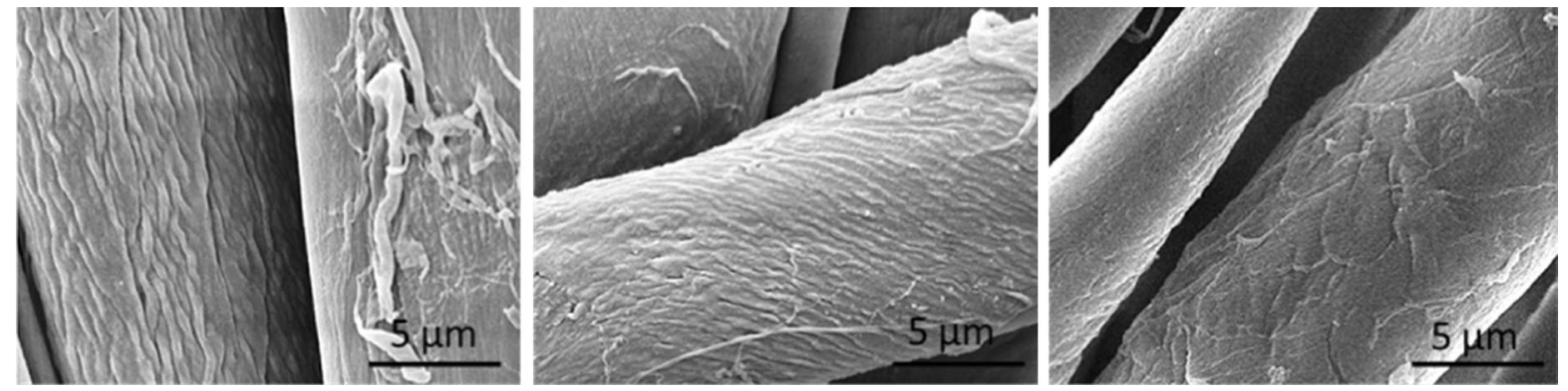

Fig. 1 SEM images of the cotton fabric before the purification by extraction

table with a set inclination angle, driven by a stepper motor with a step of 1/8, delivered by Zaber (Canada, model T-LLS105). The tested samples were fixed on the table by double-sided adhesive tape. Deionized water with a droplet volume of $10 \mu \mathrm{l}$ was placed on the sample surface. The values determined are the averages of five individual measurements (Kiuru and Alakoski 2004).

\section{Surface conductivity of the modified fabric samples}

The electrical properties of the cotton samples with a deposited network of MWCNTs were measured using a Keithley 2400C SourceMetter ${ }^{\circledR}$. The measurements were made by the four-wire method under normal conditions. The electrodes consisted of conductive silver paste from Dotite ${ }^{\circledR}$ Silver Paint D-550 (Japan), and the distance between them was $1 \mathrm{~cm}$. The acquisition of data was made by LabTrace 2.9 from Keithley.

\section{Results and discussion}

Cotton fibers are characterized by a considerable heterogeneity of their morphological laminar structure and microstructure as well as the amount, type and arrangement of natural impurities such as waxes, fats, proteins, pectins, hemicellulose and mineral lignins (Fig. 1).

This heterogeneity results from the different conditions of fiber growth and maturing, degree of fiber maturity and light accessibility. The place of origin and climatic conditions in various fiber maturing periods influence the morphological structure and microstructure of fibers, as well as the content and distribution, in various fiber layers, of natural impurities, whose total quantity amounts to 8-12\% of fiber weight. Various technological impurities (fats, dispersing agents, finishing agents and sizes) also contribute to the fiber heterogeneity. Therefore, the basic condition for decreasing the natural heterogeneity of cotton fibers and fabrics is the removal of their impurities. By using proper conditions of purifying processes, one can to some extent unify the fiber properties to provide appropriate sorption properties. This allows one to properly deposit specified substances by wet methods to obtain the expected results of the processes used, e.g., depositing CNTs or agents imparting hydrophobic properties to fibers. All the fabric samples used in ours experiments were preliminarily purified by extraction. The extraction conditions used also exert some positive influence on the microstructure of cotton fibers in terms of improving their homogeneity. The effectiveness of the method used to preliminarily purify cotton fibers was confirmed by the SEM image (Fig. 2d) showing the uncovered surface of fibers with cellulose microfibrils.

Under these conditions, it is also possible to increase and equalize the accessibility of active functional hydroxyl groups of cellulose, which is of significant importance for proper sorption processes as well as combining the chemical agents deposited, e.g., to increase the hydrophobic properties (Cunha et al. 2010). As a result, the method of sample prepurification used exerts a positive effect on the homogeneity of the MWCNTs or MTCS deposited as confirmed by the SEM images (Fig. 3) of cotton fibers with deposited MWCNTs. This is particularly visible after the removal of the dispersing agent (SDS) by rinsing in water. In the course of washing out, also nanotubes in the form of clusters freely lying on the fiber surface are removed (Fig. 3a, b), as confirmed by SEM images (Fig. 3c, d). 

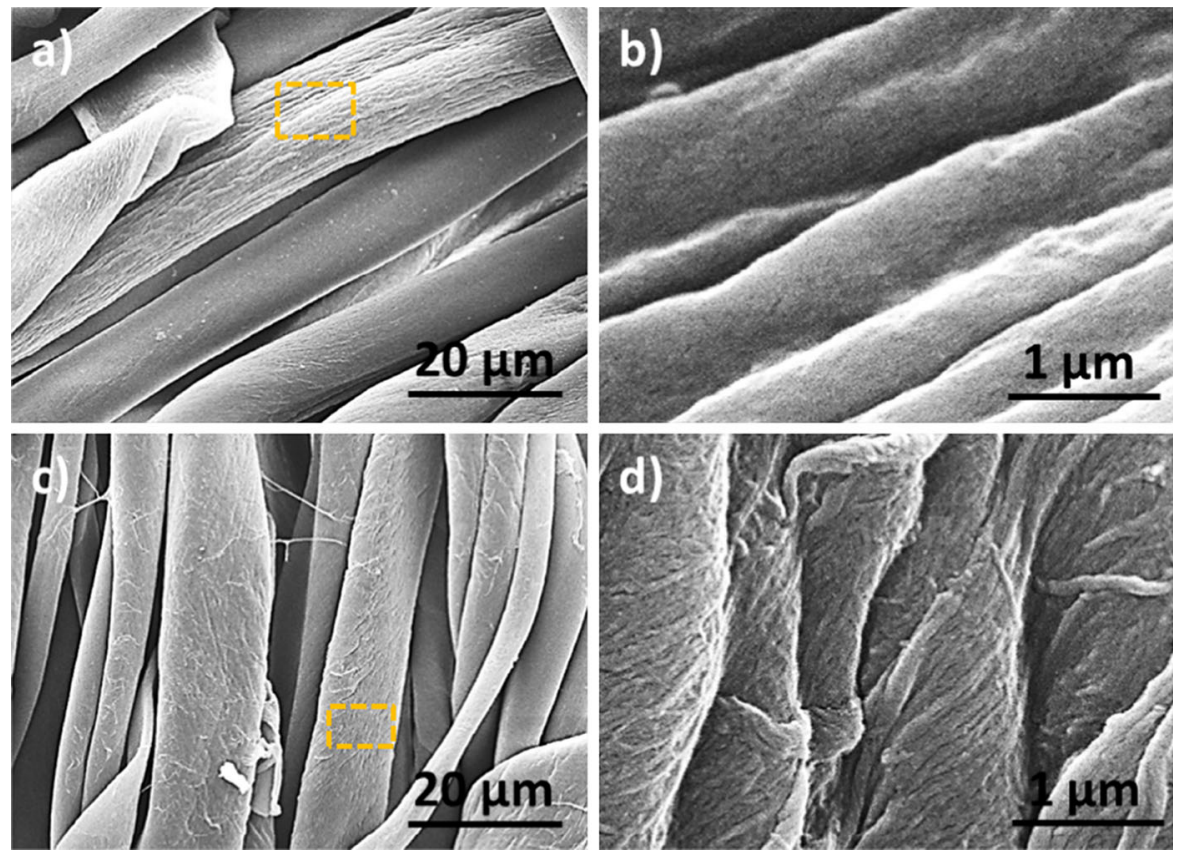

Fig. 2 SEM images of the cotton fabric: before $(\mathbf{a}, \mathbf{b})$ and after $(\mathbf{c}, \mathbf{d})$ purification by extraction
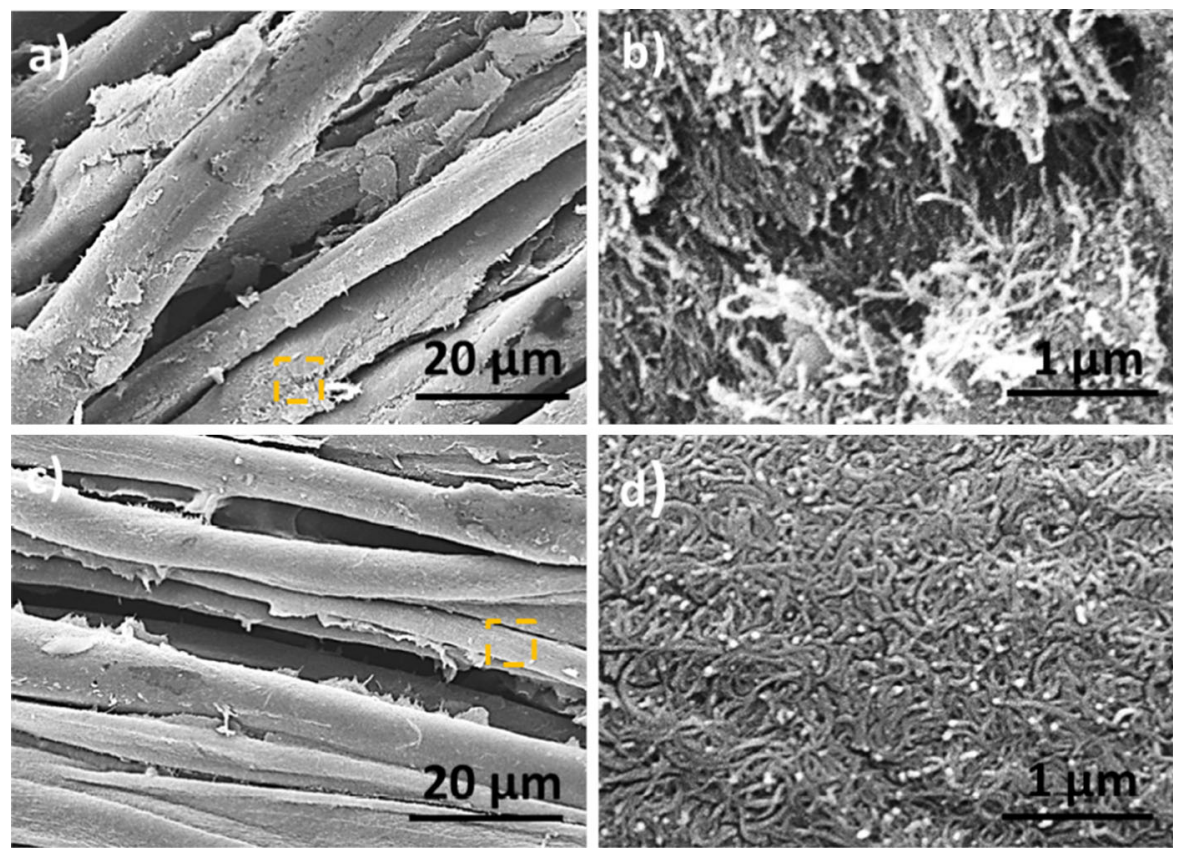

Fig. 3 SEM images of the cotton fabric containing MWCNTs: before (a), (b) and after washing out SDS (c), (d)

The surface resistance measurements performed show that the washing out of excess MWCNTs does not adversely effect on the electric properties of the tested sample. This would indicate that during the fabric padding with MWCNT solution, a percolation network is formed on the fiber surface. The removal of 
Fig. 4 EDS spectra of the cotton fabrics containing MWCNTs: before (left) and after washing out SDS (right)

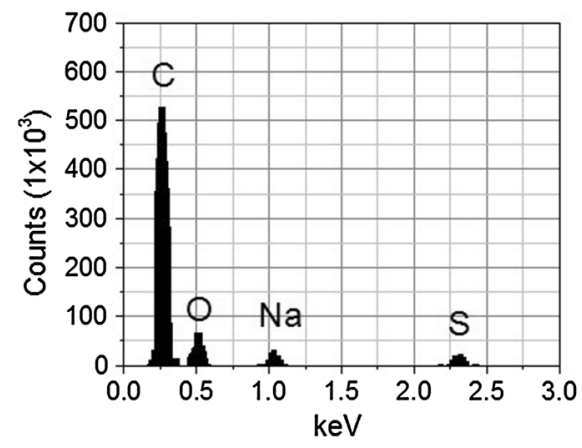

SDS is also confirmed by the EDS examination of the chemical composition of the fiber surface layer. Figure 4 shows the EDS spectra of the fabric samples with the deposited MWCNT network before and after the washing out process, where one can observe the decay of signals derived from sodium and sulfur atoms.

The hydrophobization process was carried out with the use of MTCS in liquid phase using prepurified fabrics. In order to investigate the effect of the CNT presence on cotton fibers on their hydrophobic properties, hydrophobization was carried out on both untreated cotton fabric samples and samples with deposited MWCNT layers. Besides the duration of hydrophobization and the concentration of silane (Shirgholami et al. 2013; 2011), a considerable impact on the size and shape of the structures formed on the fiber surface is exerted by the fiber's initial moisture content. Such a dependence has not been mentioned in the literature. SEM images (Figs. 5, 6) show the samples of cotton textiles hydrophobized at various initial relative humidity values (RH 0, 40 and $70 \%$ ), for untreated samples and samples containing deposited MWCNTs, respectively. The spherical nanostructures being formed consist of polymethylsilsesquioxane durably or loosely combined with the fiber surface. The rinsing of the fabrics in water using an ultrasonic washing machine removes the nanostructures chemically unbound with the fiber surface, which has no adverse effect on the hydrophobicity effect obtained.

\section{Mechanism of hydrophobization}

Many layers of water combining with the fiber surface in different ways (Fig. 7) make it difficult to talk about one simple mechanism of cotton fiber hydrophobization.
In the first stage, chlorosilane reacts with water molecules loosely combined with the cotton fiber surface. The hydrolysis of $\mathrm{Si}-\mathrm{Cl}$ bonds leads to the appearance of free silanol molecules with various numbers of silanol groups capable of condensing. Silanol molecules condense with themselves to form boundary particles in the hydrophobic solvent (toluene). On account of the presence of a great number of silanol groups, the surface energy of these groups is high, and they assume a spherical shape (Fig. 8).

Methyl groups are heading towards the solvent, while silanol groups undergo condensation, forming the PMSQ structures. The fiber devoid of water molecules in this way discloses its own hydroxyl groups capable of reacting with chlorosilane. The addition may proceed through a single bond while the remaining two reactive groups combined with silicon in the form of chlorine atoms make it possible to react with the hydroxyl groups of silane (Fig. 9).

For this reason, the depositions on the hydrophobized fiber surface consist of microstructures attached by covalent bonds and structures covering the surface thanks to electrostatic interactions or hydrogen bonds (Fig. 5). The greater the fiber moisture is, the greater the number of free silanols in the vicinity of their surface. The presence of the methyl group in silanol and the toluene medium causes silanol groups to orient toward the fiber. This creates convenient conditions for condensation with hydroxyl groups on fibers. The structures formed on the fiber surface consist of PMSQ groups, typical of silicone resins.

The nanostructures formed on the purified cellulose fiber surface with different moisture contents are presented in the SEM images (Fig. 5). On the fabric fiber surface with minimal initial water content (dry sample RH $\sim 0 \%$ ) spherical nanoobjects with 

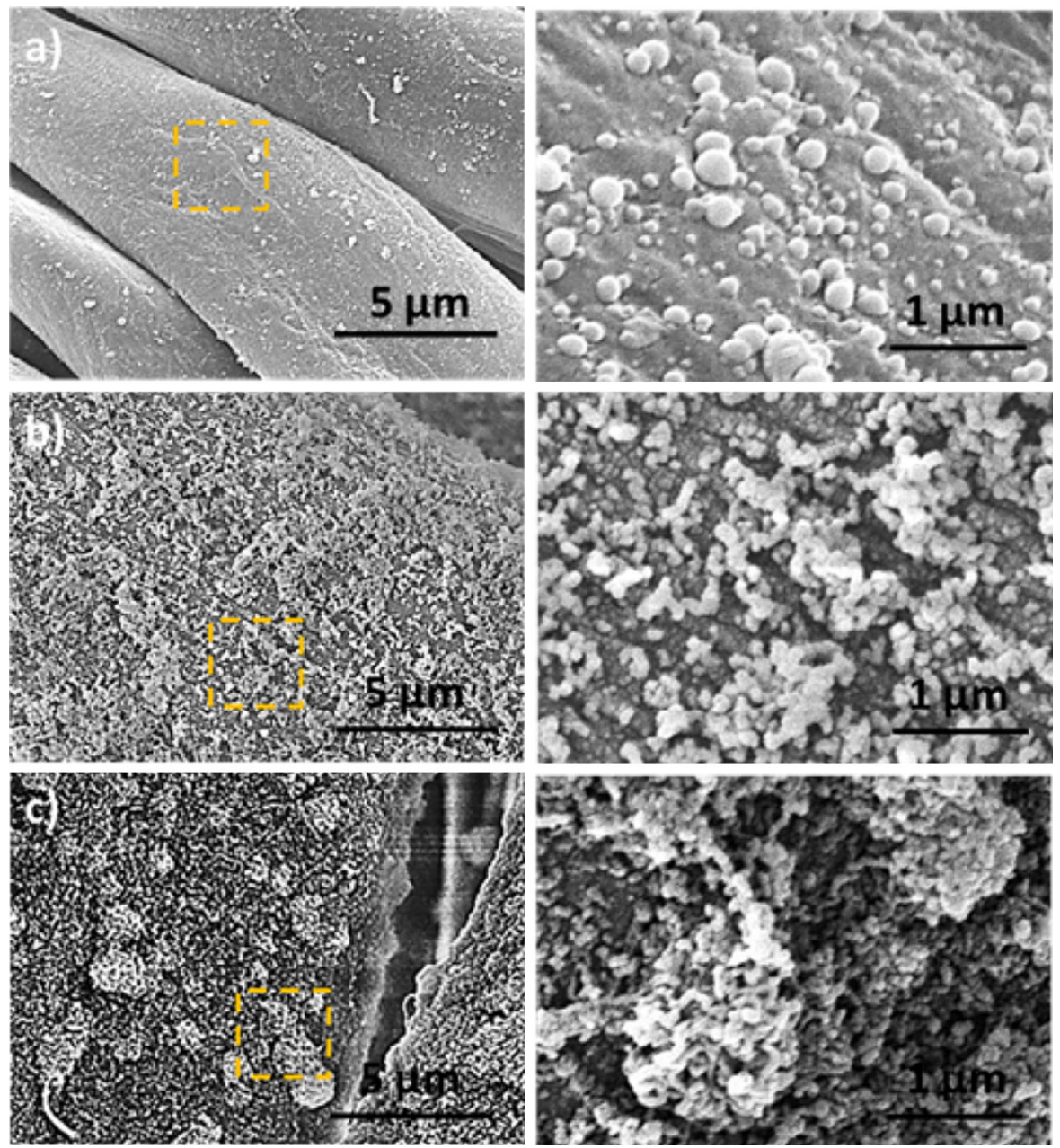

Fig. 5 SEM images of the purified cotton fabrics after hydrophobization for various humidity values: $\mathrm{RH}=0 \%(\mathbf{a}), \mathrm{RH}=40 \%$ (b), $\mathrm{RH}=70 \%(\mathbf{c})$

different sizes could be observed. With increasing moisture content of fibers, conditioned in air at $\mathrm{RH}=40 \%$, nanofibrous structures consisting of aggregated PMSQ spheres appear on their surface as shown in Fig. 6. At RH $=70 \%$ these nanofilaments are set up into spheres ("balls") (Fig. 10), with their distribution on the fiber surface being heterogeneous.

The measurements of water contact angles (WCA) and sliding angles (SA) were carried out for purified cotton fabrics and fabrics containing a layer of deposited nanotubes. The results are listed in Table 1.

In the case of cotton fabrics, their hydrophobicity increases with increasing of the initial moisture content in hydrophobized fibers. In the case of samples with initial zero moisture content, the contact angle was at a level of $144^{\circ}$, which corresponds to the so- called rose petal effect (Bhushan and Nosonovsky 2010), and thereby having the capability of maintaining a water drop on the fabric surface, even when it is turned by $180^{\circ}$ (sticky surface)(Chen et al. 2010). The sliding angle (SA) determined for this sample amounted to $22^{\circ}$. At higher initial moisture contents in hydrophobized samples, contact angles increased, reaching $156^{\circ}$ for the sample with $\mathrm{RH}=40 \%$, which is a typical value for so-called superhydrophobic surfaces, defined as the lotus leaf effect (Bixler and Bhushan 2013; Nishimoto and Bhushan 2013), causing a free water drop to roll from the fabric surface, even under conditions of a small inclination in relation to the horizontal position (slippery surface). The boundary value of the water contact angle in the case of superhydrophobic fabrics is defined as WCA 

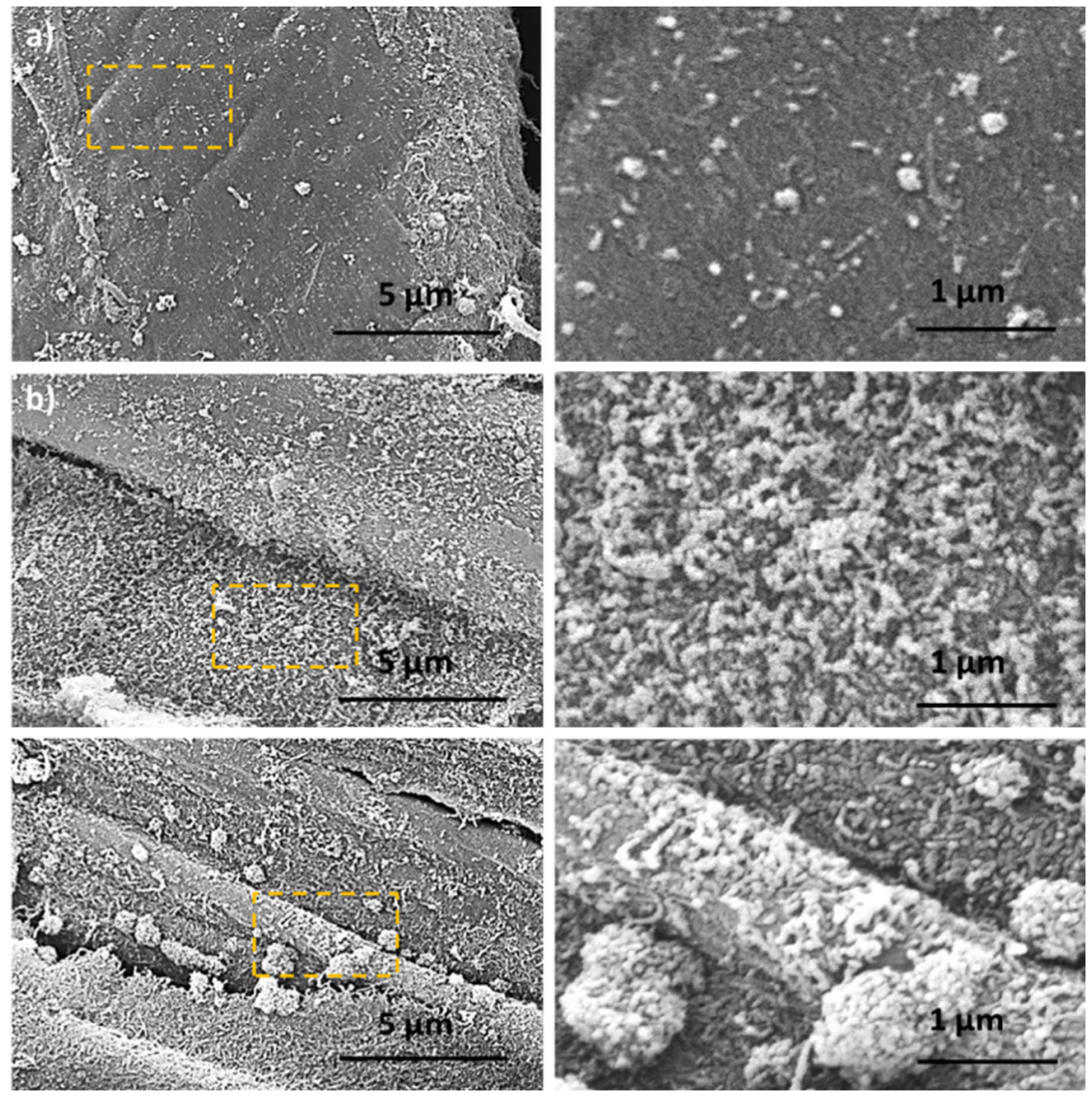

Fig. 6 SEM images of the cotton fabrics containing MWCNTs after hydrophobization for various humidity values: RH $=0 \%$ (a), $\mathrm{RH}=40 \%(\mathbf{b}), \mathrm{RH}=70 \%(\mathbf{c})$

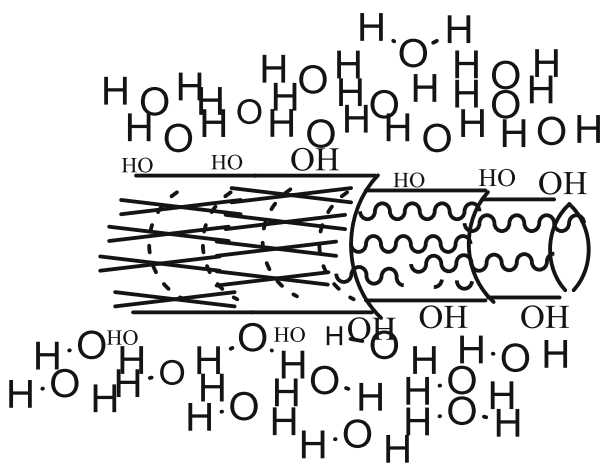

Fig. 7 Scheme of the fiber structure and layers of water around a single cotton fiber

$\alpha \geq 150^{\circ}$ (Crick and Parkin 2010; Xue et al. 2012). In the case of samples conditioned in air at $\mathrm{RH}=70 \%$, we even obtained a level of $170^{\circ}$, indicating its very high superhydrophobicity. In the case of samples with WCA over $150^{\circ}$, we also obtained very good results of sliding angles (for fabric at $\mathrm{RH}=40 \%, \mathrm{SA}=10^{\circ}$; at $\mathrm{RH}=70 \%, \mathrm{SA}=6^{\circ}$ ). The values of WCA and SA of the hydrophobized cotton fabric samples are listed in Table 2.

The results obtained prove that the initial water content in the tested sample is an important parameter in the formation process of PMSQ structures on the cotton fabric surface, starting from single objects through their set up as nanofilaments to a mixture of various nanostructures in the shape of "balls."

It was of interest to observe a great similarity between the PMSQ structures on the purified cotton fiber surfaces $(\mathrm{CO})$ and those preliminarily covered with a conductive network of multiwall CNTs $(\mathrm{CO}+\mathrm{MWCNT}$ ) (Figs. 5 and 6). Both types of samples were conditioned and hydrophobized under 
Fig. 8 Scheme of the formation of micro- and nanospheres in toluene solution

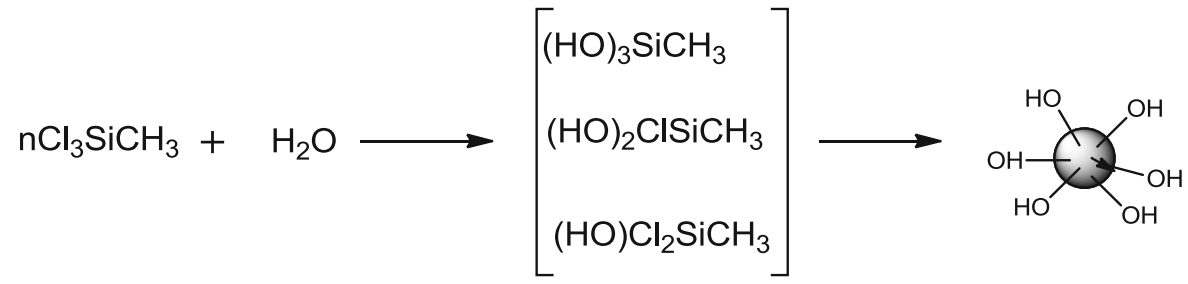

Fig. 9 Stages of the hydrophobization of the cotton fiber surface with deposited micro- and nanospheres
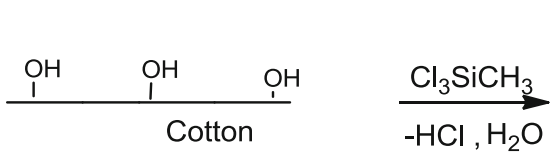

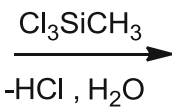

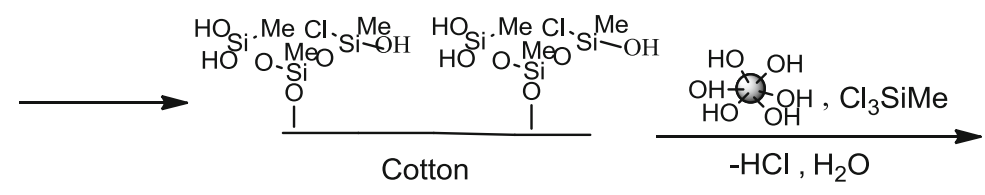
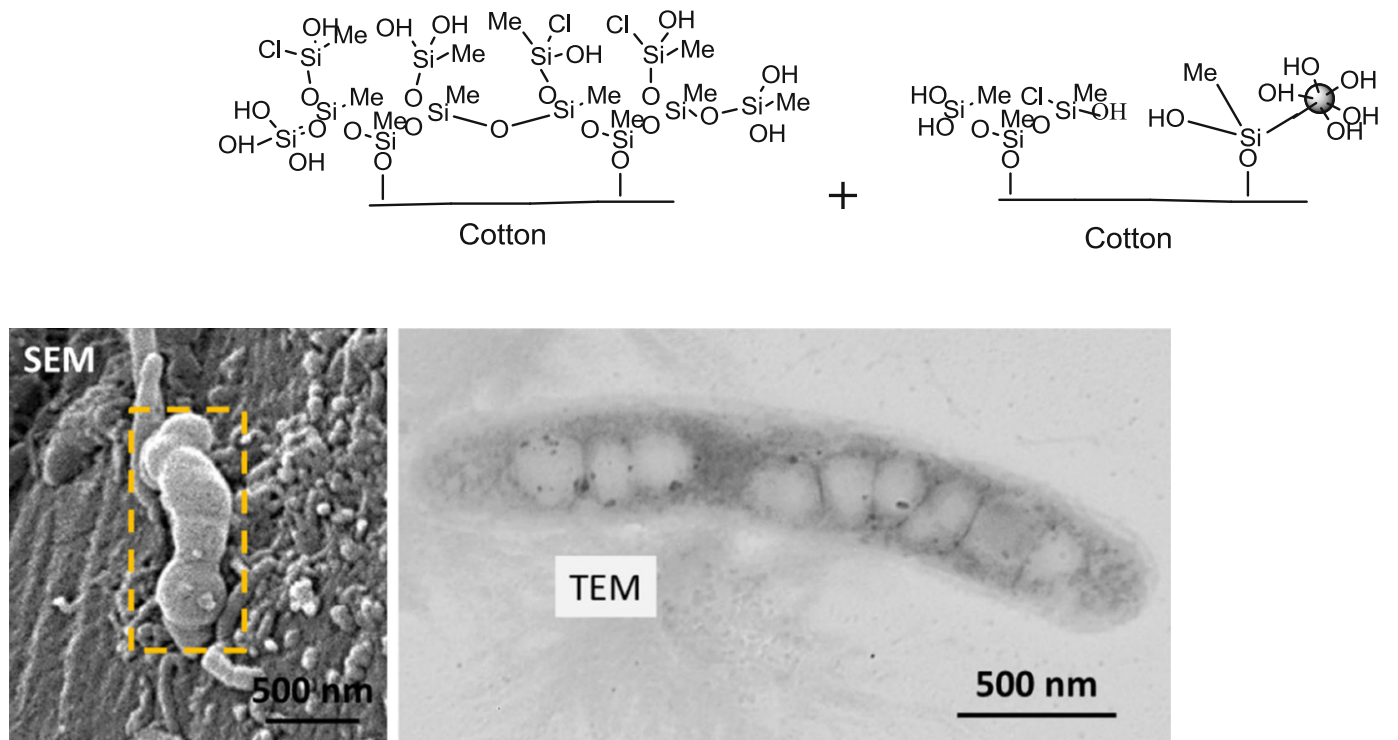

TEM

$500 \mathrm{~nm}$

Fig. 10 TEM image of the PMSQ nanofilament structure on the surface of cotton fiber $(\mathrm{RH}=70 \%)$

the same conditions. The results obtained indicate that the multiwall CNTs present on the fiber surface have no significant impact on the shape of the nanostructures formed, and thereby, as one may assume, on the reaction mechanism between MTCS and functional (hydroxyl) groups (Figs. 8 and 9) on the tested fiber/ fabric surfaces (Fig. 6). Despite the fact that there is a lack of visible differences in the surface morphologies of the samples compared, the WCAs determined for cotton fabrics with multiwall CNTs assume higher values, which indicates their stronger hydrophobic properties (Table 2). For the $\mathrm{CO}+\mathrm{MWCNT}$ sample conditioned at $\mathrm{RH}=70 \%$, WCA amounted to $170^{\circ}$, and the SA determined was $6^{\circ}$. In the case of these samples, one could observe the strongest lotus leaf effect.

Based on the tests performed, one can conclude that the hydrophobicity effect obtained is exclusively 
Table 1 Water contact angles (WCAs) and sliding angles (SA) determined for initial samples of purified cotton fabric (CO) and the cotton fabric containing multiwall carbon nanotubes $(\mathrm{CO}+\mathrm{MWCNTs})$

\begin{tabular}{lcc}
\hline Sample & WCA $\left(^{\circ}\right)$ & SA $\left(^{\circ}\right)$ \\
\hline $\mathrm{CO}$ & 0 & - \\
$\mathrm{CO}+$ MWCNTs & 134 & \\
& & \\
\hline
\end{tabular}

determined by the PMSQ structures formed on the fiber surface that are dependent on the fiber moisture level. These nanostructures also impart the effect of nanoroughness, especially visible in the case of fibers/ fabrics with the highest initial moisture content, corresponding to $\mathrm{RH}=70 \%$ (ball structures).

The electrical properties of the hydrophobized cotton fabrics were also tested to assess the effect of their surface modification on changes in electric conductivity. From the results obtained presented in Fig. 11a, for the samples of the cotton fabric with deposited of multiwall CNTs, it follows that with an increasing number of paddings, and thereby the amount of MWCNTs deposited, the fabric resistance decreases and consequently its surface conductivity is increased. A linear character (Fig. 11a-4) and the lowest resistance $\left(0.71 \mathrm{k} \Omega / \mathrm{cm}^{2}\right)$ were obtained for the samples padded four times. In the case of the samples padded once, twice and three times, the resistance determined amounts of $394.14,16.75$ and $3.54 \mathrm{k} \Omega$ / $\mathrm{cm}^{2}$, respectively. On account of the best electrical properties of the sample padded four times, its resistance was measured after the modification with MTCS depending on the initial RH (Fig. 11b) for RH values of 0,40 and $70 \%$; the resistance values obtained amounted to $1.11,6.33$ and $8.60 \mathrm{k} \Omega / \mathrm{cm}^{2}$, respectively. Hence, the hydrophobization caused a decrease in the fiber conductivity, which depends on the water content in fibers and thereby on the density of the nanostructures formed on the cotton surface.

\section{Conclusions}

The examination of the cotton fabric hydrophobization by the wet method in a waterless medium with the use of MTCS has shown that one of the significant parameters of the hydrophobization process is the initial water content in the analyzed fiber/fabric samples. It has been shown that by changing the fabric moisture content one can control the mechanism of MTCS treatment (silylation) and thereby control the size and shape of the silicon nanostructures under formation. An increase in humidity facilitates the formation of nanofilament structures that impart superhydrophobic properties to the modified cotton fabrics (lotus effect). These nanostructures also impart nanoroughness on the surface of cotton fiber, supporting the superhydrophobicity obtained, especially visible in the

Table 2 WCA and SA values determined for: purified cotton fabric (CO) and cotton fabric containing multiwall carbon nanotubes $(\mathrm{CO}+\mathrm{MWCNTs})$ subjected to hydrophobization for various initial relative humidity values $(\mathrm{RH})$

\begin{tabular}{|c|c|c|c|c|}
\hline \multirow[t]{2}{*}{ RH (\%) } & \multicolumn{2}{|c|}{ WCA $\left(^{\circ}\right)$} & \multicolumn{2}{|c|}{ SA $\left(^{\circ}\right)$} \\
\hline & $\mathrm{CO}$ & $\mathrm{CO}+\mathrm{MWCNTs}$ & $\mathrm{CO}$ & $\mathrm{CO}+\mathrm{MWCNTs}$ \\
\hline 0 & 144 & 155 & 22 & 13 \\
\hline 40 & 156 & 164 & 10 & 8 \\
\hline 70 & 163 & 170 & 7 & 6 \\
\hline
\end{tabular}




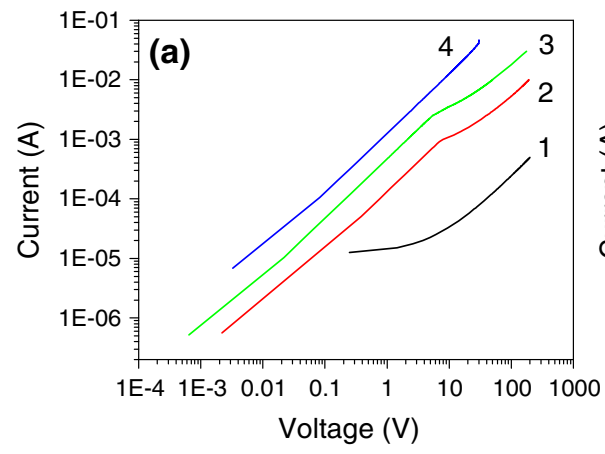

Fig. 11 Current-voltage characteristics I (V): for various numbers of paddings (a); curves 1, 2, 3 and 4 denote a sample padded with MWCNTs once, twice, three and four times,

case of fibers/fabrics with the highest initial water content, conditioned in air at $\mathrm{RH}=70 \%$ (ball structures). To obtain a uniform deposition of the hydrophobizing compound and CNTs on the surface of fiber and distribution of PMSQ nanostructures, it is important to remove all impurities, especially fats and waxes, which can be successfully accomplished with the use of extraction. As a result, one can obtain a homogeneous fiber surface and a significant increase in the number of accessible hydroxyl groups. It has been shown that the hydrophobicity level obtained is exclusively determined by the PMSQ structures formed on the surface of the sample depending on the initial water content in the fibers. Based on the results obtained, it was possible to propose the mechanism of the silylation of cotton fibers explaining the superhydrophobic properties imparted to these fibers. The basic result of the hydrophobization of cotton fabrics with a deposited MWCNT network is the partial change in their electric properties depending on the water content in fibers (tenfold decrease in conductivity) in relation to initial fabric samples (initial resistance below $1 \mathrm{k} \Omega / \mathrm{cm}^{2}$ ), with no changes in the fabric hydrophobicity.

Acknowledgments The authors would like to thank the following for financial support of this work: the National Science Centre (DEC-2011/03/B/ST8/06126) and Przemyslaw Sowinski from CMMS PAS for the TEM picture.

Open Access This article is distributed under the terms of the Creative Commons Attribution License which permits any use, distribution, and reproduction in any medium, provided the original author(s) and the source are credited.

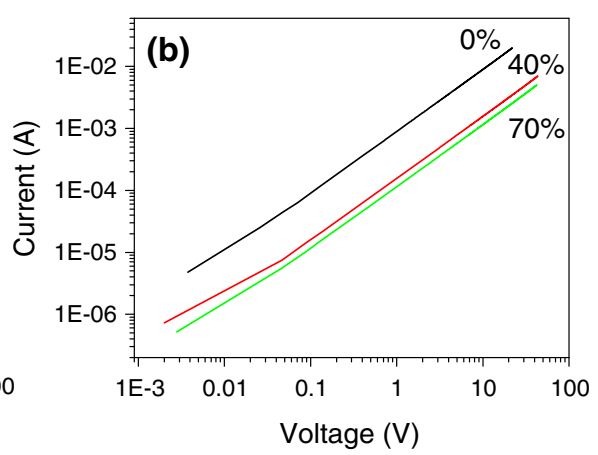

respectively; for various humidity values for a sample padded four times with MWCNTs (b)

\section{References}

Alimohammadi F, Gashti MP, Shamei A (2012) A novel method for coating of carbon nanotube on cellulose fiber using 1,2,3,4-butanetetracarboxylic acid as a cross-linking agent. Prog Org Coat 74:470-478. doi:10.1016/j.porgcoat.2012. 01.012

Alimohammadi F, Parvinzadeh Gashti F, Shamei A (2013) Functional cellulose fibers via polycarboxylic acid/carbon nanotube composite coating. J Coat Technol Res 10:123-132. doi:10.1007/s11998-012-9429-3

Al-Shabib W, Habibi D, Xie ZH, Zhao XL (2012) Identifying smart conducting materials for Wi-Fi electromagnetic interference shielding Asia-Pac Int Sym Ele 741-744

Andersson P et al (2002) Active matrix displays based on allorganic electrochemical smart pixels printed on paper. Adv Mater 14:1460-1464. doi:10.1002/1521-4095(20021016)14: 20<1460:Aid-Adma1460>3.0.Co;2-S

Artus GRJ, Jung S, Zimmermann J, Gautschi HP, Marquardt K, Seeger S (2006) Silicone nanofilaments and their application as superhydrophobic coating. Adv Mater 18:2758-2762. doi:10.1002/adma.200502030

Bhushan B, Nosonovsky M (2010) The rose petal effect and the modes of superhydrophobicity. Philos T R Soc A 368:4713-4728. doi:10.1098/rsta.2010.0203

Bixler GD, Bhushan B (2013) Fluid drag reduction and efficient self-cleaning with rice leaf and butterfly wing bioinspired surfaces Nanoscale 5:7685-7710. doi:10.1039/C3nr01710a

Chen XQ, Liu YY, Lu HF, Yang HR, Zhou XA, Xin JH (2010) In-situ growth of silica nanoparticles on cellulose and application of hierarchical structure in biomimetic hydrophobicity. Cellulose 17:1103-1113. doi:10.1007/s10570010-9445-3

Crick CR, Parkin IP (2010) Preparation and characterisation of super-hydrophobic surfaces. Chem-Eur J 16:3568-3588. doi:10.1002/chem.200903335

Cunha AG et al (2010) Preparation of highly hydrophobic and lipophobic cellulose fibers by a straightforward gas-solid reaction. J Colloid Interf Sci 344:588-595. doi:10.1016/j. jcis.2009.12.057 
Ding XF, Zhou SX, Gu GX, Wu LM (2011) A facile and largearea fabrication method of superhydrophobic self-cleaning fluorinated polysiloxane/TiO2 nanocomposite coatings with long-term durability. J Mater Chem 21:6161-6164. doi:10.1039/C0jm04546b

Fadeev AY, McCarthy TJ (2000) Self-assembly is not the only reaction possible between alkyltrichlorosilanes and surfaces: monomolecular and oligomeric covalently attached layers of dichloro- and trichloroalkylsilanes on silicon. Langmuir 16:7268-7274. doi:10.1021/La000471z

Feng L et al (2002) Super-hydrophobic surfaces: from natural to artificial. Adv Mater 14:1857-1860. doi:10.1002/adma. 200290020

Fischer T, Wetzold N, Elsner H, Kroll L, Hubler AC (2011) Carbon nanotube areas: printed on textile and paper substrates regular paper. Nanomater Nanotechno 1:18-23

Gao LC, McCarthy TJ (2009) Wetting 101 degrees. Langmuir 25:14105-14115. doi:10.1021/La902206c

Gao LC, McCarthy TJ, Zhang X (2009) Wetting and superhydrophobicity. Langmuir 25:14100-14104. doi:10.1021/ La903043a

Hamdani STA, Potluri P, Fernando A (2013) Thermomechanical behavior of textile heating fabric based on silver coated polymeric yarn. Materials 6:1072-1089. doi:10.3390/Ma6031072

Hench LL, West JK (1990) The sol-gel process. Chem Rev 90:33-72. doi:10.1021/Cr00099a003

Hertleer C, Rogier H, Vallozzi L, Van Langenhove L (2009) A textile antenna for off-body communication integrated into protective clothing for firefighters. IEEE T Antenn Propag 57:919-925. doi:10.1109/Tap.2009.2014574

Hock CW, Harris M (1940) Microscopic examination of cotton fibers in cuprammonium hydroxide solutions. J Res Natl Bur Stand 24:743-748

Hosono E, Fujihara S, Honma I, Zhou HS (2005) Superhydrophobic perpendicular nanopin film by the bottom-up process. J Am Chem Soc 127:13458-13459. doi:10.1021/ $\mathrm{Ja} 053745 \mathrm{j}$

Jost K, Perez CR, McDonough JK, Presser V, Heon M, Dion G, Gogotsi Y (2011) Carbon coated textiles for flexible energy storage. Energy Environ Sci 4:5060-5067. doi:10.1039/ C1EE02421C

Kennedy TF, Fink PW, Chu AW, Champagne NJ, Lin GY, Khayat MA (2009) Body-worn e-textile antennas: the good, the low-mass, and the conformal. IEEE T Antenn Propag 57:910-918. doi:10.1109/Tap.2009.2014602

Kiuru M, Alakoski E (2004) Low sliding angles in hydrophobic and oleophobic coatings prepared with plasma discharge method. Mater Lett 58:2213-2216. doi:10.1016/j.matlet. 2004.01.024

Lee GY, Psychoudakis D, Chen CC, Volakis JL (2011) omnidirectional vest-mounted body-worn antenna system for UHF operation. IEEE Antenn Wirel Pr 10:581-583. doi:10.1109/ Lawp.2011.2158381

Li XM, Reinhoudt D, Crego-Calama M (2007) What do we need for a superhydrophobic surface? A review on the recent progress in the preparation of superhydrophobic surfaces. Chem Soc Rev 36:1529. doi:10.1039/B602486f
Liu Y, Gorgutsa S, Santato C, Skorobogatiy M (2012) Flexible, solid electrolyte-based lithium battery composed of LiFePO4 cathode and Li4Ti5O12 anode for applications in smart textiles. J Electrochem Soc 159:A349-A356. doi:10. 1149/2.020204jes

Nishimoto S, Bhushan B (2013) Bioinspired self-cleaning surfaces with superhydrophobicity, superoleophobicity, and superhydrophilicity. Rsc Adv 3:671-690. doi:10.1039/C2ra21260a

Park K-Y, Lee S-E, Kim C-G, Han J-H (2006) Fabrication and electromagnetic characteristics of electromagnetic wave absorbing sandwich structures. Compos Sci Technol 66:576-584. doi:10.1016/j.compscitech.2005.05.034

Parvinzadeh Gashti M, Almasian A (2013) UV radiation induced flame retardant cellulose fiber by using polyvinylphosphonic acid/carbon nanotube composite coating. Compos B Eng 45:282-289. doi:10.1016/j.compositesb. 2012.07.052

Schoen DT, Schoen AP, Hu LB, Kim HS, Heilshorn SC, Cui Y (2010) High speed water sterilization using one-dimensional nanostructures. Nano Lett 10:3628-3632. doi:10. 1021/N1101944e

Shirgholami MA, Shateri Khalil-Abad M, Khajavi R, Yazdanshenas ME (2011) Fabrication of superhydrophobic polymethylsilsesquioxane nanostructures on cotton textiles by a solution-immersion process. J Colloid Interf Sci 359:530-535. doi:10.1016/j.jcis.2011.04.031

Shirgholami MA, Shateri-Khalilabad M, Yazdanshenas ME (2013) Effect of reaction duration in the formation of superhydrophobic polymethylsilsesquioxane nanostructures on cotton fabric. Text Res J 83:100-110. doi:10.1177/ 0040517512444335

Vaisman L, Wagner HD, Marom G (2006) The role of surfactants in dispersion of carbon nanotubes. Adv Colloid Interfac 128:37-46. doi:10.1016/j.cis.2006.11.007

Xie X, Hu LB, Pasta M, Wells GF, Kong DS, Criddle CS, Cui Y (2011) Three-dimensional carbon nanotube-textile anode for high-performance microbial fuel cells. Nano Lett 11:291-296. doi:10.1021/N1103905t

Xu QF, Wang JN, Smith IH, Sanderson KD (2009) Superhydrophobic and transparent coatings based on removable polymeric spheres. J Mater Chem 19:655-660. doi:10. 1039/B812659c

Xue ZX, Liu MJ, Jiang L (2012) Recent developments in polymeric superoleophobic surfaces. J Polym Sci Pol Phys 50:1209-1224. doi:10.1002/Polb.23115

Yang L, Rida A, Vyas R, Tentzeris MM (2007) RFID tag and RF structures on a paper substrate using inkjet-printing technology. IEEE T Microw Theory 55:2894-2901. doi:10. 1109/Tmtt.2007.909886

Yang Y-L, Chuang M-C, Lou S-L, Wang J (2010) Thick-film textile-based amperometric sensors and biosensors. Analyst 135:1230-1234. doi:10.1039/B926339J

Zha J, Roggendorf H (1991) Sol-gel science, the physics and chemistry of sol-gel processing, Ed. by C. J. Brinker and G. W. Scherer, Academic Press, Boston 1990, xiv, 908 pp., bound-ISBN 0-12-134970-5 Adv Mater 3: 522 doi:10. 1002/adma.19910031025 